Article

\title{
Multi-Time-Scale Simulation Tool for Renewable Energy Integration Analysis in Distribution Circuits
}

\author{
Pisitpol Chirapongsananurak ${ }^{1}$ and Surya Santoso ${ }^{2, *}$ \\ 1 Department of Electrical Engineering, Chulalongkorn University, Bangkok 10330, Thailand; \\ pisitpol.c@chula.ac.th \\ 2 Department of Electrical and Computer Engineering, The University of Texas at Austin, Austin, \\ TX 78712, USA \\ * Correspondence: ssantoso@mail.utexas.edu; Tel.: +1-512-471-3371
}

Academic Editors: Josep M. Guerrero and Chien-Hung Liu

Received: 10 February 2017; Accepted: 11 April 2017; Published: 13 April 2017

\begin{abstract}
This paper develops a distribution circuit multi-time-scale simulation tool for applications in wind turbine and photovoltaic (PV) integration analysis. The proposed simulation tool developed in MATLAB ${ }^{\mathrm{TM}}$ includes several distribution circuit components, such as voltage sources, distribution lines, transformers, loads, capacitor banks, wind turbines and PVs. Each equipment consists of three models for simulations in three different time scales, i.e., steady-state, electromechanical transient and electromagnetic transient models. Therefore, the proposed tool is able to perform a long-term simulation involving power system phenomena spreading across time scales. The test circuits employed to perform multi-time-scale simulation in this paper are modified from the IEEE four-node test feeder. The simulation scenarios include wind speed and solar irradiance ramp up and down; a capacitor bank is energized and de-energized; and a single-line-to-ground fault occurs and clears itself. The simulation results show that the proposed tool is capable of evaluating power system phenomena spread across time scales.
\end{abstract}

Keywords: distributed power generation; multi-time-scale simulation; power system modeling; power system simulation; solar power generation; wind power generation

\section{Introduction}

Increasing penetration of renewable energy sources (RESs), such as wind and solar, raises concerns regarding their impacts on a power system. Although wind and solar power generation can reduce peak load demand and losses in a distribution circuit [1], these RESs may cause problems in a circuit, such as voltage regulation [1-3], flicker [3,4], reverse power flow [1,5], short-circuit current contribution $[2,3,6,7]$, protection coordination [2], capacitor switching transients and harmonics distortion $[2,3,8,9]$.

Power system simulation tools have been widely used to analyze the potential impacts of wind turbines and photovoltaics (PVs). These tools can help evaluate power system phenomena, such as voltage regulation, fault study, switching transients and harmonics analysis. The simulation tools can also be used to perform the feasibility studies of operating wind turbines and PVs in a distribution system [10,11], analyze the stability of a distribution system with wind and solar power [12,13], determine the maximum penetration or hosting capacity of wind turbines and PVs [14-16], evaluate the performance of wind turbine and PV control methods $[17,18]$ and investigate the effects of energy storage systems [19-21] and reactive power sources, such as capacitor banks, static var compensators (SVCs), static synchronous compensators (STATCOMs) $[19,22,23]$ and dynamic voltage restorers (DVRs) [24] to a distribution system having wind and PV generators. 
Power system phenomena can be categorized according to their time scales into three types, i.e., electromagnetic transient, electromechanical transient and quasi-steady-state phenomena. Because the time scales of these phenomena vary from fractions of a cycle to a few hours, each existing simulation tool is designed for only one specific time scale. Therefore, these existing single-time-scale tools are not able to analyze long-term power system phenomena spreading across different time scales. In order to allow power system analysis involving phenomena spreading across time scales, this paper aims to develop a multi-time-scale simulation tool by integrating the existing steady-state, electromechanical transient and electromagnetic transient simulation tool. The objective of this paper involves developing the approaches to select which single-time-scale model to use for each disturbance and the approaches to switch between two single-time-scale models. In addition, due to the unbalanced nature of distribution circuits, the proposed tool employs distribution circuit models with all three phases represented.

This paper is organized as follows: Section 2 describes the characteristics of each type of power system phenomena. Section 3 explains the proposed multi-time-scale simulation approach including the development and integration of the single-time-scale simulation models. Section 4 presents the case studies used to demonstrate the multi-time-scale simulation approach, including test circuits, simulation results and the discussion, followed by the conclusion in Section 5 .

\section{Power System Phenomena}

There are three types of phenomena in power systems (categorized based on their time scales): electromagnetic transient, electromechanical transient and quasi-steady-state phenomena. The typical time scales of these phenomena are shown in Figure 1 [25].

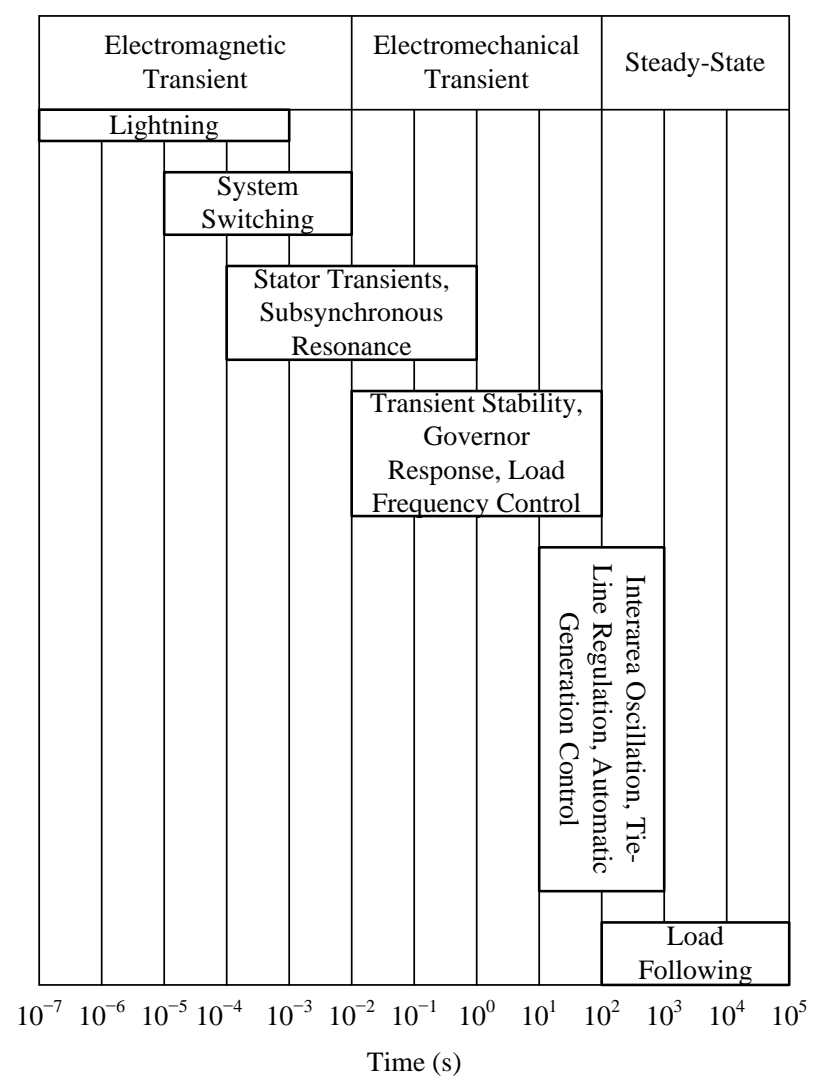

Figure 1. Time scales of power system phenomena. 
The time scale of electromagnetic transient phenomena (effects of capacitor switching and lightning strikes on voltage and current in power systems) is usually in the range of microseconds and milliseconds because these phenomena involve the exchange of energy between electric and magnetic fields (inductors and capacitors) present in power systems. Thus, in order to analyze these phenomena, the effect of inductors and capacitors (distribution lines, transformers, capacitor banks and electrical machine windings) in transient conditions must be included in the simulation models. However, the dynamics of mechanical components in power systems, such as the inertia of electric machines, have much longer time scales and hence can be discarded.

The time scale of electromechanical transient phenomena (effects of short-circuit faults, transient stability analysis, inertial response study and frequency response study) is usually in the range from hundreds of milliseconds up to tens of seconds because these phenomena involve the exchange of energy between electrical and mechanical components in the systems (inductors, capacitors and inertia) due to a mismatch between power generation and load consumption. Therefore, the dynamics of electromechanical components, e.g., inertia of rotating mass in electrical machines, must be included in the electromechanical transient model. However, the dynamics associated with the electromagnetic transients in distribution lines, transformers, capacitor banks and stators of electrical machines may be neglected.

The time scale of quasi-steady-state phenomena (effects of voltage regulation, flicker, voltage unbalance, harmonics, load demand variation, wind speed variation and solar irradiance variation) is usually in the range of several minutes or longer. These phenomena are analyzed by steady-state simulation tools neglecting all dynamics, both electromagnetic and electromechanical transient, of the power systems.

\section{Multi-Time-Scale Simulation Approach}

This section describes the single-time-scale models (steady-state, electromechanical transient and electromagnetic transient) of distribution circuits employed in this paper, as well as the approach for integrating the single-time-scale models to perform a multi-time-scale simulation.

\subsection{Modeling}

The proposed distribution circuit multi-time-scale simulation tool implemented in the $\mathrm{MATLAB}^{\mathrm{TM}}$ environment consists of steady-state, electromechanical transient and electromagnetic transient models of distribution circuit equipment (distribution lines, transformers and capacitor banks), wind turbines and PVs. The models of the equipment used in this paper follow the well-established modeling approaches described in [26-30].

\subsubsection{Models of Distribution Circuit Equipment}

Typical distribution circuits consist of the following equipment: distribution lines, transformers and capacitor banks. Each equipment model consists of its steady-state, electromechanical transient and electromagnetic transient models.

1. Steady-state model: Since all transient dynamics (both electromechanical and electromagnetic transients) are not included in a steady-state simulation, each distribution circuit equipment can be modeled by a fixed impedance or admittance in a frequency domain (phasor form). In a distribution network, the equipment is connected to each other. Therefore, the fixed impedance and admittance models for each equipment are combined and form a nodal admittance matrix [27].

2. Electromechanical transient model: A distribution network (distribution lines, transformers and capacitors) can be represented by electrical components, i.e., resistors, inductors and capacitors. As a consequence, the distribution network contains only dynamics associated with electromagnetic transients and has no dynamics associated with electromechanical transients 
because the distribution network does not have any mechanical components. Because the electromagnetic transient dynamics are discarded in an electromechanical transient simulation, the electromechanical transient model of a distribution network is simply its steady-state model.

3. Electromagnetic transient model: In this paper, the electromagnetic transient program (EMTP) [26] is used to simulate a distribution network in an electromagnetic transient condition. According to this approach, the trapezoidal method is used to integrate the differential equations representing each distribution circuit element in a time domain. Each algebraic equation obtained from the integration is rearranged to describe the relation between the node voltage and injected current at each terminal of the equipment (nodal equation). These nodal equations are then combined to form a matrix. This resulting matrix is similar to the nodal admittance matrix representing the distribution network in a steady-state condition.

\subsubsection{Models of a Wind Turbine}

Wind turbines can be categorized into four technologies: a fixed-speed wind turbine (FSWT), a wide-slip wind turbine (WSWT), a doubly-fed induction generator (DFIG) wind turbine and a full converter wind turbine. The model for each technology is described as follows.

The model of FSWTs and WSWTs consists of two major parts, i.e., an induction generator and mechanical components (blade inertia, generator inertia, a gearbox and shafts). The model of each part consists of steady-state, electromechanical transient and electromagnetic transient models for simulations in three different time scales.

1. Steady-state model: In a quasi-steady-state condition, FSWTs and WSWTs can be represented by a steady-state model obtained by discarding all transient dynamics in the induction generator and mechanical components. In other words, all derivative terms in the model are set to zero. As a consequence, the induction generator can be represented by three fixed impedances in a frequency domain, i.e., positive-, negative- and zero-sequence impedances. In order to obtain the generator slip, the generator torque is calculated from wind speed. For the model of WSWTs, the external rotor resistance is set so that the power output of the wind turbines is equal to the rated power when wind speed is higher than the rated speed.

2. Electromechanical transient model: In order to simulate induction generators of FSWTs and WSWTs in an electromechanical transient simulation, this paper uses the reduced model of induction generators obtained by disregarding the transients in the stator windings. Hence, only the transients in the rotor windings are modeled. The generator model consists of three equivalent circuits (for positive, negative and zero sequences) in a frequency domain. The inertia of the generator and the blades of FSWTs and WSWTs are represented by a two-mass model. For WSWTs, the external rotor resistance is adjusted by using a PI controller in order to obtain the rated power output when wind speed is above the rated speed.

3. Electromagnetic transient model: A complete model of induction generators considering both stator and rotor transients is employed in an electromagnetic transient simulation. The blade and generator speeds are assumed to be constant because of the short simulation time, so the rotating mass maintains essentially the same speed. For the WSWT model, the external rotor resistance, in addition to the speed of the generator and the blades, is also assumed to be constant due to the short simulation time.

Since DFIG and full converter wind turbines employ converters to control their real and reactive power output [30], the characteristic of the wind turbines follows the converter control approaches. Therefore, DFIG and full converter wind turbines can be represented by a converter model with its control. In this paper, the converter is assumed to produce only positive-sequence current at the fundamental frequency. In other words, the harmonic currents are neglected. The model of DFIG and full converter wind turbines consists of three models, i.e., steady-state, electromechanical transient and electromagnetic transient models. 
1. Steady-state model: Since DFIG and full converter wind turbines employ a converter to control the real and reactive power output, the wind turbines can be represented by a constant power load in a steady-state condition. The real power of the constant power load model is obtained from the real power control set point (maximum power point tracking curve), while the reactive power of the constant power load model is obtained from the reactive power control set point. Note that the power in the constant power load model is negative because the wind turbines generate power.

2. Electromechanical transient model: For an electromechanical transient simulation, DFIG and full converter wind turbines can be represented by a current source model. The current of the current source model is controlled by the same approach as the converter control. Since the real and reactive power output of the wind turbines are independently controlled by the converter, the current of the current source model is separated into the components on $q$ - and $d$-axes. The $q$-axis current component is used to control the real power output of the wind turbines, while the d-axis current component is used to control the reactive power output of the wind turbines. Each current component is controlled by a PI controller similar to the PI controller employed by the converter control.

3. Electromagnetic transient model: Because the time scale of the converter control of DFIG and full converter wind turbines is close to the border between the electromechanical and electromagnetic transient phenomena, the wind turbine model for electromechanical transient simulation is identical to the model for electromechanical transient simulation, i.e., the wind turbines are represented by a current source model with two PI controllers for real and reactive power controls.

\subsubsection{Models of PV}

PVs are connected to the grid via inverters, which can be used to control the real and reactive power output of the PV. Therefore, PV can be represented by an inverter model with its control. The inverter model consists of three models for electromagnetic transient, electromechanical transient and steady-state simulations. These models are similar to the models of DFIG and full converter wind turbines.

\subsection{Model Selection and Switching between Models}

The proposed multi-time-scale simulation tool consists of three single-time-scale models, i.e., steady-state, electromechanical transient and electromagnetic transient models. Currently, the proposed simulation tool is used for offline analyses, so the capability of the simulation tool to detect the types of disturbances occur in the distribution circuit and select which single-time-scale models to use is not required. Before the multi-time-scale simulation starts, the timings to use each single-time-scale model are pre-selected according to the types of each disturbance as described in Table 1. Figure 2 shows the sample time line for a multi-time-scale simulation with multiple disturbances. The sequence of the single-time-scale models used in the multi-time-scale simulation in Figure 2 is summarized in Figure 3. As shown in Figure 3, the multi-time-scale simulation involves switching between two single-time-scale models, i.e., between the steady-state and electromechanical transient models, and between the steady-state and electromagnetic transient models. In order to smoothly switch between two single-time-scale models, the energy stored in each storage element (inductor, capacitor, inertia and spring) in both single-time-scale models must be the same. This condition suggests that the voltages, currents, torques and rotational speeds of every element must be the same before and after switching between the models. This condition also implies that when the simulation switches from the steady-state model to the electromechanical or electromagnetic transient models or vice versa, the electromechanical or electromagnetic transient models must rest in a steady-state condition, i.e., the electromechanical or electromagnetic transients have already died down. 
Table 1. Selected models for sample disturbances.

\begin{tabular}{cc}
\hline Sample Disturbance & Selected Model \\
\hline Wind and solar variation & Steady-state \\
Fault & Electromechanical transient \\
Capacitor switching & Electromagnetic transient \\
\hline
\end{tabular}

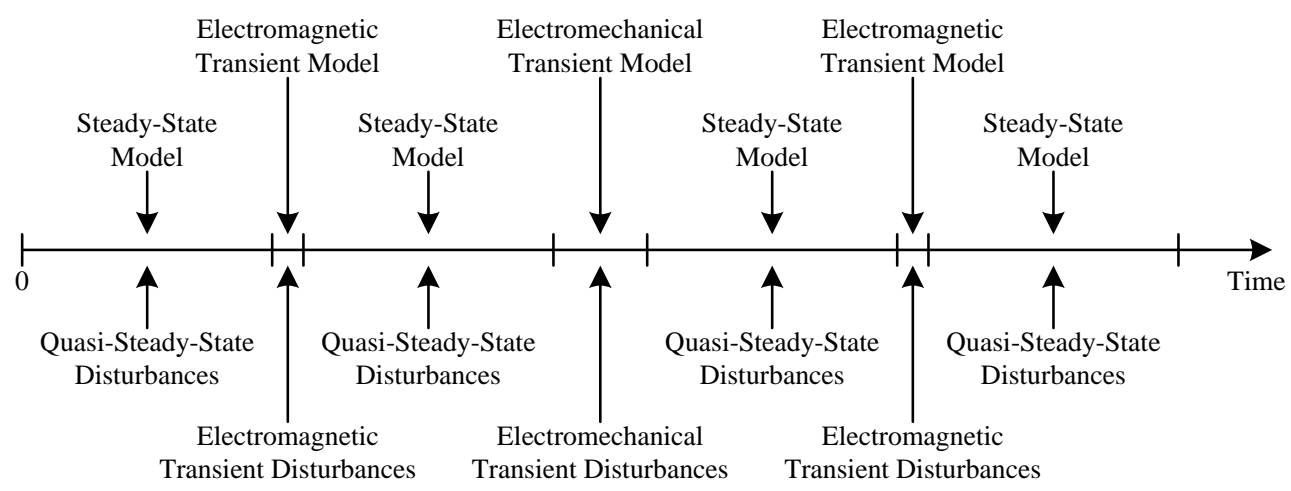

Figure 2. Time line for a multi-time-scale simulation.

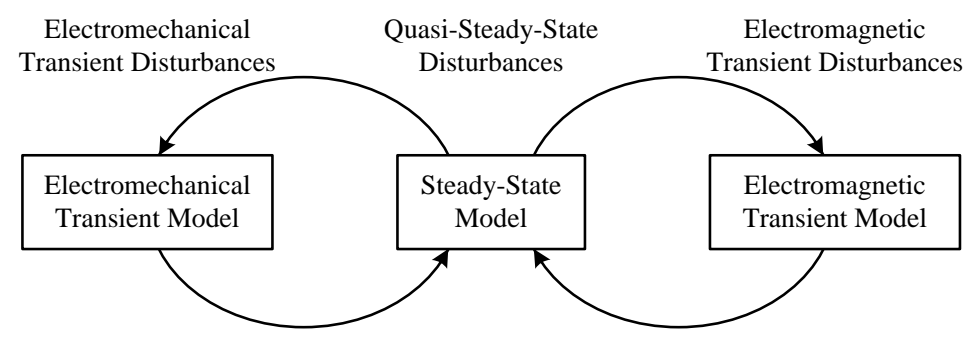

Figure 3. Multi-time-scale simulation diagram.

The simulation is performed by the steady-state model when quasi-steady-state disturbances such as wind speed and solar irradiance variations occur. Wind speed and solar irradiance variations are considered as quasi-steady-state disturbances because the typical resolution of wind speed and solar irradiance data is much longer than the transient period.

The electromechanical transient model is used to simulate electromechanical transient disturbances such as short-circuit faults. The simulation starts by performing a steady-state simulation at the time right before the disturbance occurs. Then, the steady-state results are used to initialize the electromechanical transient model. Thus, the electromechanical transient simulation begins from the last quasi-steady-state condition before the disturbance occurs. When the disturbance occurs, the electromechanical transient model is used to obtain the simulation results in the electromechanical transient period. When the power system reaches steady-state, the electromechanical transient results at the last time step are used to initialize the steady-state model so that the system conditions before and after switching are the same.

For electromagnetic transient disturbances such as capacitor switching, the steady-state model is used first to obtain results at the last quasi-steady-state condition before the disturbance occurs. Then, the steady-state results are used to initialize the electromagnetic transient model. Therefore, the electromagnetic transient simulation starts from the quasi-steady-state condition at the time right before the disturbance occurs. When the disturbance occurs, the electromagnetic transient model is used to simulate results in the electromagnetic transient period. After the electromagnetic transient dies down and the system reaches a steady-state condition, the electromagnetic transient results are 
used to initialize the steady-state model so that the system conditions before and after switching are the same.

\section{Simulation of Distribution Circuits}

This section describes the case studies for the multi-time-scale simulations including the test circuits, simulation results and discussions.

\subsection{Test Circuits}

There are two test circuits used in this paper: the four-node test circuit for wind turbine simulations and the three-node test circuit for PV simulations. Both test circuits are modified from the IEEE four-node test feeder [31] to include a wind turbine and PV.

\subsubsection{Four-Node Test Circuit for Wind Turbine Simulation}

The one-line diagram of the four-node test circuit for wind turbine simulation is shown in Figure 4. This test circuit has five components, i.e., an infinite bus, two unbalanced distribution lines, a transformer, a capacitor bank and a wind farm. The capacitor bank is installed at the low voltage side of the transformer. The ratings of the wind farm's generators are $1.8 \mathrm{MVA}$ and $690 \mathrm{~V}$. Figure 5 shows the ten-minute resolution wind speed data obtained from National Renewable Energy Laboratory (NREL) [32] used in this paper.

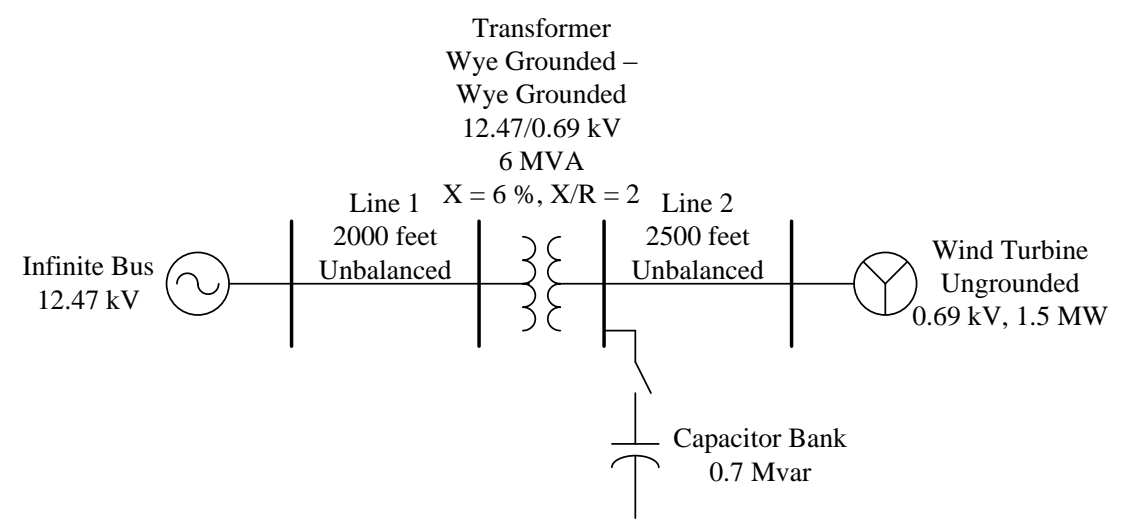

Figure 4. One-line diagram of the four-node test circuit for wind turbine simulations. [33]

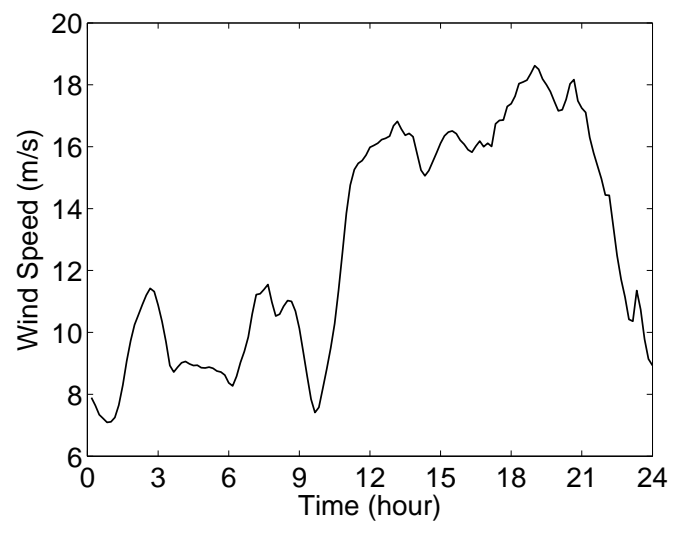

Figure 5. Wind speed for the multi-time-scale simulation [33].

This paper performs a multi-time-scale simulation of the wind turbine test circuit for a 24 -h period. Wind speed changes every ten minutes during the entire simulation. When the simulation starts at 
12:00 a.m., the capacitor bank is out of service. The capacitor bank is switched on and off at 8:00 a.m. and 10:00 p.m., respectively. At 2:00 p.m., a temporary bolted single-line-to-ground fault occurs on Phase A of the high voltage side of the transformer. The fault clears itself in 0.1 seconds.

\subsubsection{Three-Node Test Circuit for PV Simulation}

Figure 6 shows the one-line diagram of the three-node test circuit for PV simulation. This test circuit consists of an infinite bus, an unbalanced four-wire distribution line, a transformer, a capacitor bank, single-phase loads and a PV. The capacitor bank is connected to the high voltage side of the transformer; the loads are connected to the low voltage side of the transformer; and the PV is connected to Phases A and B of the low voltage side of the transformer. This paper uses the one-second resolution solar irradiance data obtained from Electric Power Research Institute (EPRI) [34] shown in Figure 7.

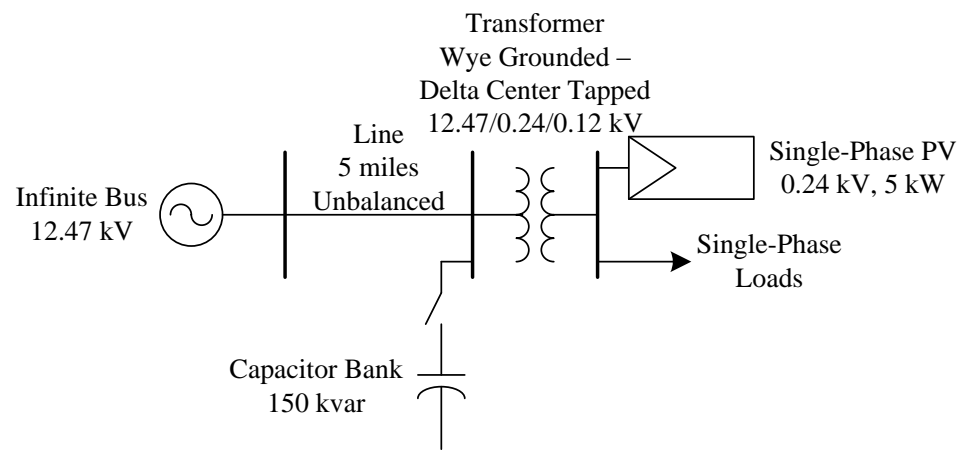

Figure 6. One-line diagram of the three-node test circuit for PV simulations.

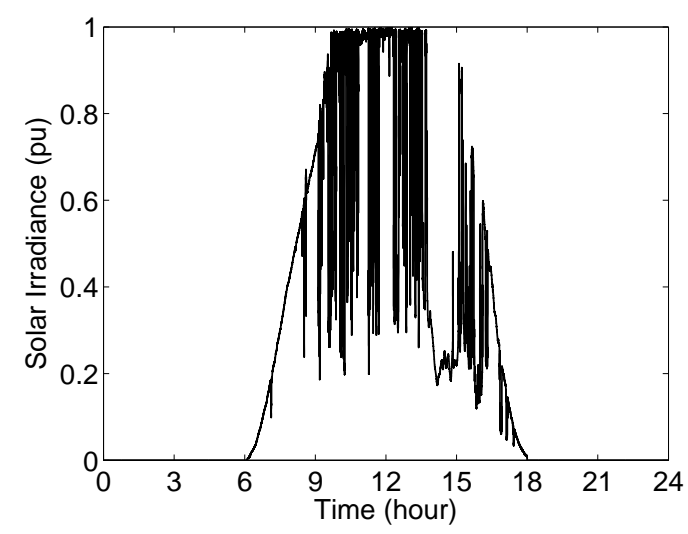

Figure 7. Solar irradiance for the multi-time-scale simulation.

The multi-time-scale simulation of the PV test circuit is performed for a 24-h period. During the entire simulation, solar irradiance changes every one second. The capacitor bank is in service when the simulation starts at 12:00 a.m. The capacitor bank is switched off at 8:00 a.m. and switched on again at 4:00 p.m. A temporary bolted single-line-to-ground fault occurs on Phase A of the high voltage side of the transformer at 12:00 p.m. The fault clears itself in $0.1 \mathrm{~s}$.

\subsection{Simulation Results and Discussion}

The distribution circuit multi-time-scale simulations are performed in MATLAB ${ }^{\mathrm{TM}}$ for a 24-h period. Four test cases are considered in this paper: simulations for a wind farm consisting of an FSWT, a wind farm consisting of a WSWT, a wind farm consisting of a DFIG or full converter wind turbine and a PV. 


\subsubsection{Case 1: Wind Farm Consisting of an FSWT}

This case simulates the four-node test circuit with a wind farm consisting of an FSWT. When wind speed changes, the multi-time-scale simulation tool employs the steady-state model because the resolution of wind speed data is much longer than the power system transient period. At 8:00 a.m., the capacitor is turned on, so the simulation tool switches from the steady-state model to the electromagnetic transient model since the capacitor energizing is an electromagnetic transient phenomenon. After the transient dies down and the circuit reaches a new steady-state condition, the steady-state model is used again. When the fault occurs at 2:00 p.m., the simulation tool switches to the electromechanical transient model because the fault dynamics is in the electromechanical transient time scale. As soon as the circuit reaches a steady-state condition after the fault is cleared, the tool switches back to use the steady-state model again. The tool switches to the electromagnetic transient model again when the capacitor is turned off at 10:00 p.m. and switches back to employ the steady-state model when a steady-state condition is reached. The steady-state and transient voltages at the wind turbine are described in Figure 8. Note that the steady-state voltages in Figure 8a are the results of the steady-state simulation; the transient voltages in Figure $8 \mathrm{c}$ (when time $>0$ ) are obtained from the electromechanical transient simulation; and the transient voltages in Figure $8 \mathrm{~b}, \mathrm{~d}$ (when time $>0$ ) are produced by the electromagnetic transient simulation.

The variations of the steady-state voltages at the wind turbine between 12:00 a.m. and 8:00 a.m., between 8:00 a.m. and 10:00 p.m. and between 10:00 p.m. and 12:00 a.m. (Figure 8a) result from the voltage drops across the distribution lines and transformer caused by the currents flowing through them. Note that the variations of the line and transformer currents result from the fluctuation of the wind power. At 8:00 a.m., the steady-state voltages at the wind turbine significantly rise since the capacitor bank is turned on. However, the wind turbine steady-state voltages dramatically drop when the capacitor bank is turned off (10:00 p.m.).

At 8:00 a.m., the capacitor is switched on. As a consequence, the voltage oscillation occurs (Figure $8 \mathrm{~b}$ ) due to the mismatch between the voltage at the capacitor location before and after switching. The voltage transient dies down after a few cycles because of the resistive element in the circuit. After the capacitor is turned on, the voltage magnitudes at the wind turbine slightly rise.

At 2:00 p.m., the temporary single-line-to-ground fault emerges on Phase A of the high voltage side of the transformer. Therefore, Phase A wind turbine voltage immediately falls as presented in Figure $8 \mathrm{c}$. The figure also shows that Phase $\mathrm{B}$ and $\mathrm{C}$ voltages at the wind turbine, however, rise when the single-line-to-ground fault occurs. After the temporary fault clears itself within $0.1 \mathrm{~s}$, the wind turbine voltages return to their initial values.

At 10:00 p.m., the capacitor bank is switched off. As a result, the voltage magnitudes at the wind turbine slightly drop (Figure 8d). The figure also shows that small high-frequency transients emerge when the capacitor is turned off due to the fact that the capacitor bank is not de-energized at the exact current zero-crossing. 


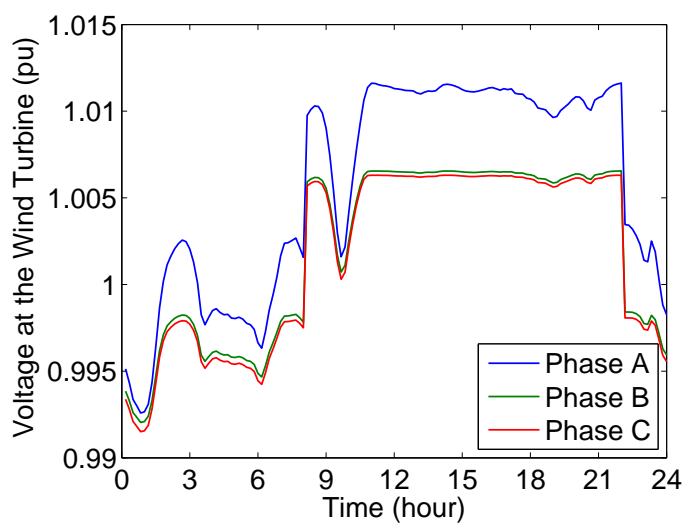

(a)

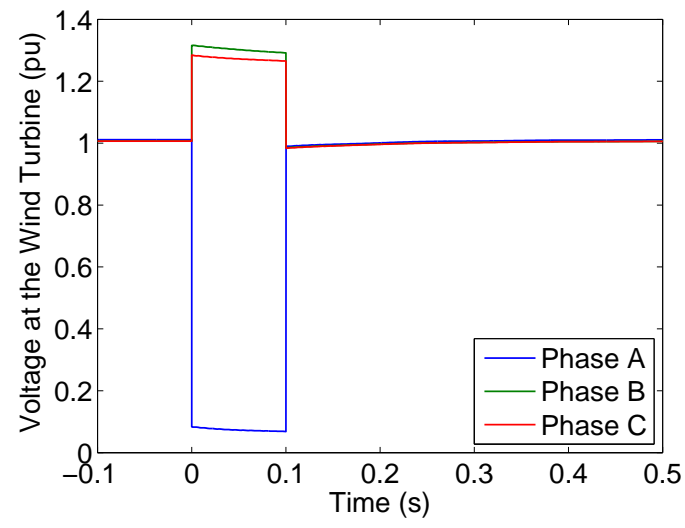

(c)

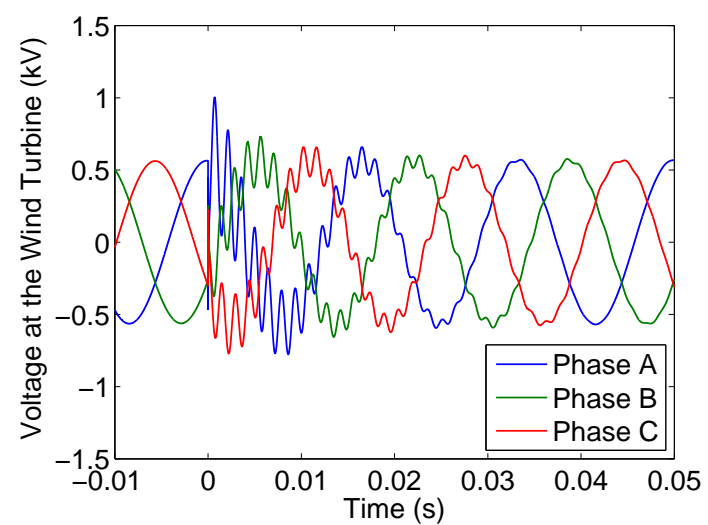

(b)

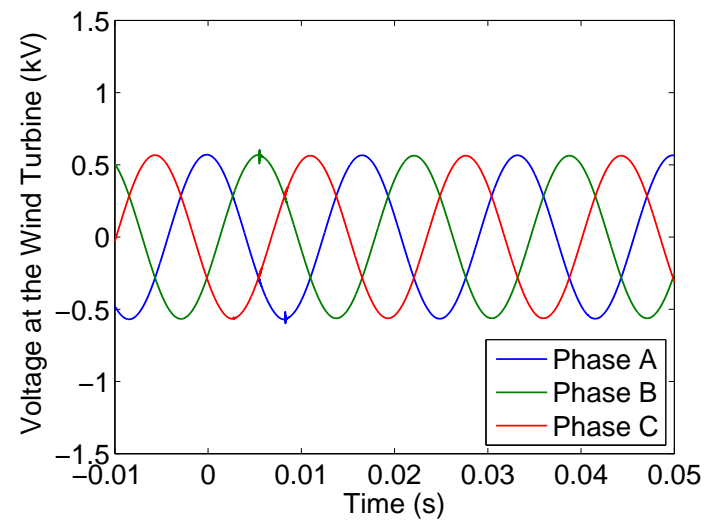

(d)

Figure 8. Voltages at the fixed-speed wind turbine (FSWT) for Case 1. (a) Steady-state voltages at the FSWT; (b) transient voltages at the FSWT when the capacitor bank is turned on at 8:00 a.m.; (c) transient voltages at the FSWT when the fault occurs at 2:00 p.m.; (d) transient voltages at the FSWT when the capacitor bank is turned off at 10:00 p.m. [33]

\subsubsection{Case 2: Wind Farm Consisting of a WSWT}

The multi-time-scale simulation of a wind farm consisting of a WSWT in quasi-steady-state, electromechanical transient and electromagnetic transient conditions is considered in this case study. This multi-time-scale simulation scenario is similar to the scenario for the FSWT simulation. Figure 9 shows the steady-state and transient voltages at the wind turbine.

Like the wind turbine voltages for the FSWT simulation, the steady-state voltages at the wind turbine between 12:00 a.m. and 8:00 a.m., between 8:00 a.m. and 10:00 p.m. and between 10:00 p.m. and 12:00 a.m. vary due to the change of the wind power as presented in Figure 9a. Note that the wind turbine produces rated power output from 11:00 a.m. to 10:00 p.m. because wind speed is above the rated speed. As a result, the voltages at the wind turbine are constant. The wind turbine voltages increase and decrease when the capacitor bank is switched on at 8:00 a.m. and off at 10:00 p.m., respectively. In addition, the electromagnetic transient voltage oscillation occurs when the capacitor bank is turned on at 8:00 a.m., as can be seen from Figure 9b. According to Figure 9c, Phase A wind turbine voltage instantly decreases when the single-line-to-ground fault occurs on Phase A at 2:00 p.m. However, Phase B and C wind turbine voltages increase. When the capacitor bank is turned off at 10:00 p.m., there are small high-frequency transients in the wind turbine voltage profile at the capacitor switching time (Figure 9d). 


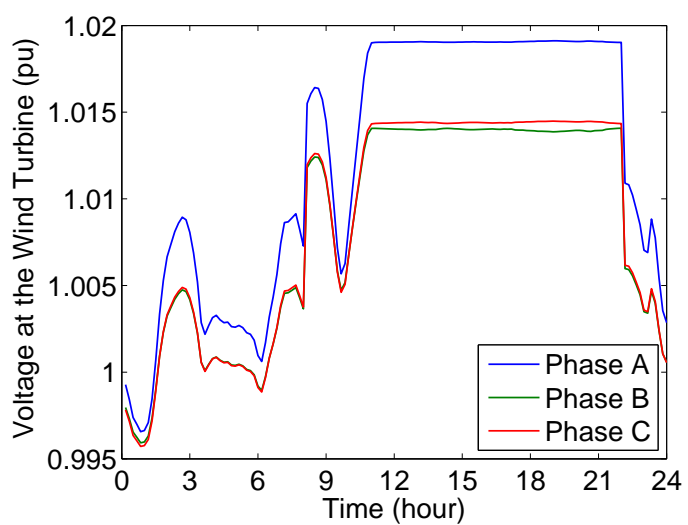

(a)

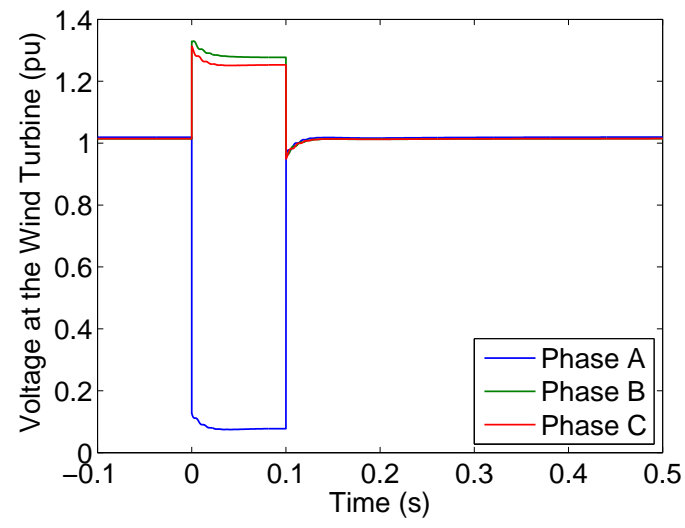

(c)

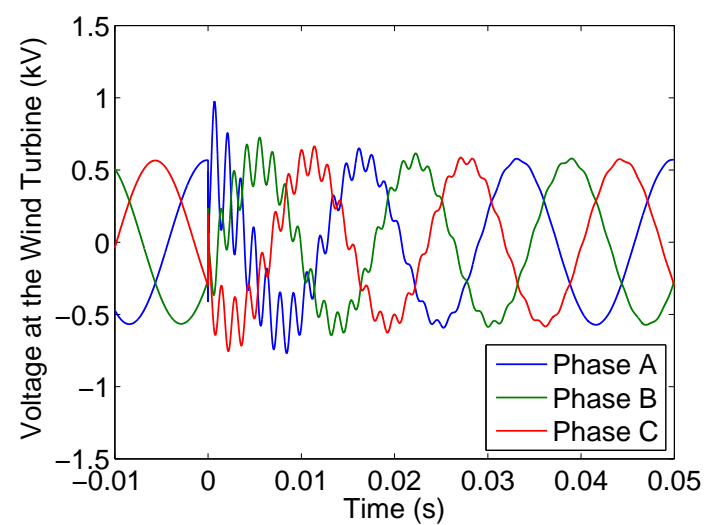

(b)

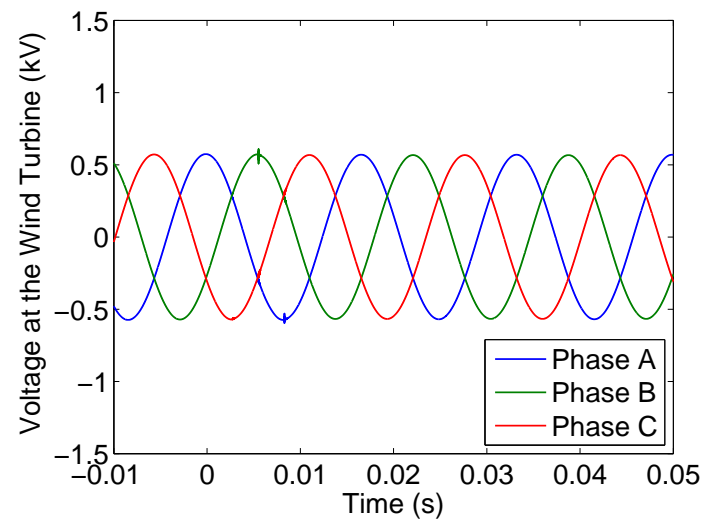

(d)

Figure 9. Voltages at the wide-slip wind turbine (WSWT) for Case 2. (a) Steady-state voltages at the WSWT; (b) transient voltages at the WSWT when the capacitor bank is turned on at 8:00 a.m.; (c) transient voltages at the WSWT when the fault occurs at 2:00 p.m.; (d) transient voltages at the WSWT when the capacitor bank is turned off at 10:00 p.m. [33]

\subsubsection{Case 3: Wind Farm Consisting of a DFIG or Full Converter Wind Turbine}

This case study considers a wind farm consisting of DFIG and full converter wind turbines in quasi-steady-state, electromechanical transient and electromagnetic transient conditions. This simulation scenario is similar to the scenarios for an FSWT and a WSWT. Figure 10 illustrates the steady-state and transient voltages at the wind turbine.

Similar to the results from the FSWT and WSWT simulations, the change of wind speed causes the variation of the steady-state voltages at the wind turbine between 12:00 a.m. and 8:00 a.m., between 8:00 a.m. and 10:00 p.m. and between 10:00 p.m. and 12:00 a.m., as presented in Figure 10a. However, the steady-state voltages at the wind turbine are flat between 11:00 a.m. and 10:00 p.m. because the wind turbine produces rated power output when wind speed is above the rated speed. The steady-state voltages rise at 8:00 a.m. because the capacitor bank is turned on at that time. However, the steady-state voltages drop at 10:00 p.m. because the capacitor bank is turned off.

Figure 10b illustrates the electromagnetic transient voltage oscillation due to the capacitor bank being energized at 8:00 a.m. Figure 10c shows that Phase A voltage at the wind turbine suddenly drops when the single-line-to-ground fault occurs on Phase A. However, Phase B and C voltages at the wind turbine rise. Because the capacitor bank is not de-energized at the exact current zero-crossing, small transients occur at the capacitor switching time (10:00 p.m.), as illustrated in Figure 10d. 


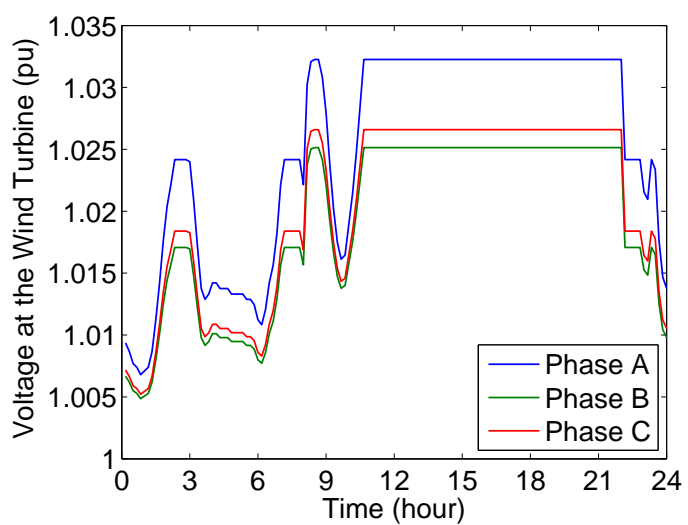

(a)

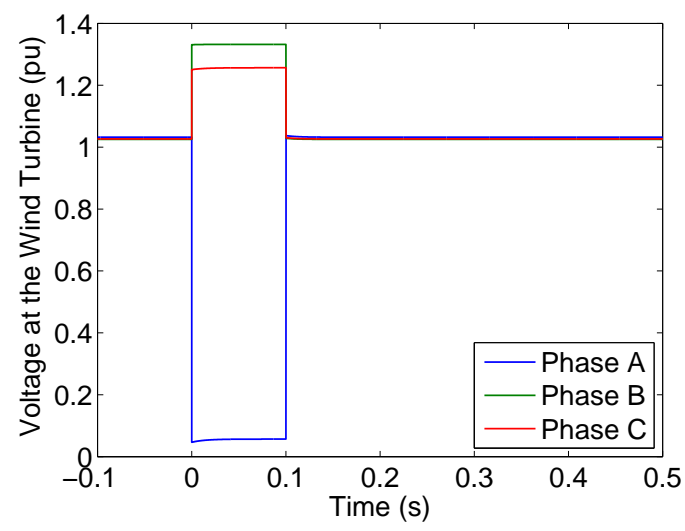

(c)

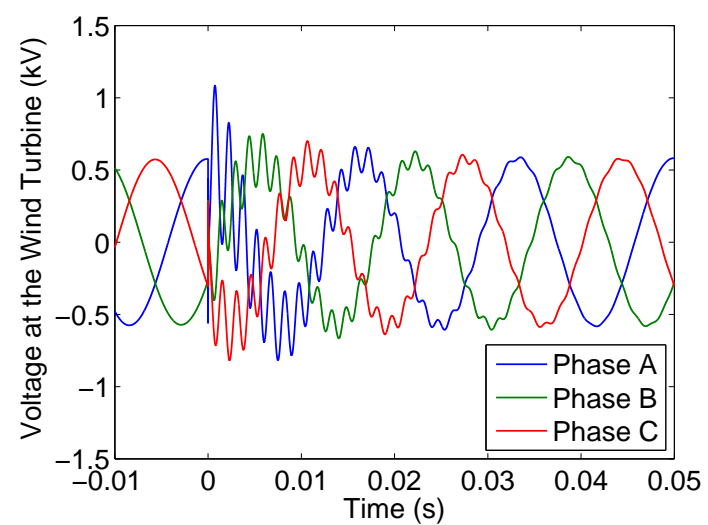

(b)

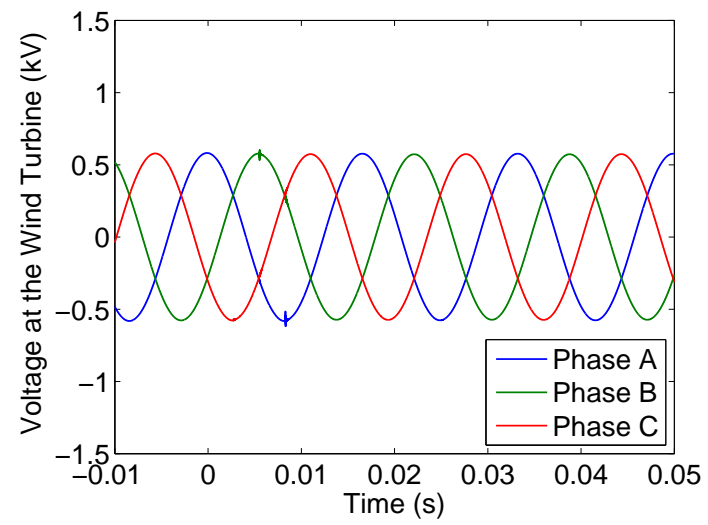

(d)

Figure 10. Voltages at the wind turbine for Case 3. (a) Steady-state voltages at the wind turbine; (b) transient voltages at the wind turbine when the capacitor bank is turned on at 8:00 a.m.; (c) transient voltages at the wind turbine when the fault occurs at 2:00 p.m.; (d) transient voltages at the wind turbine when the capacitor bank is turned off at 10:00 p.m.

\subsubsection{Case 4: PV}

This case performs a multi-time-scale simulation of the three-node test circuit with a PV. The proposed multi-time-scale simulation tool employs the steady-state model when solar irradiance changes. When the capacitor bank is switched on (8:00 a.m.) and off (4:00 p.m.), the proposed simulation tool switches to the electromagnetic transient model. However, the proposed tool switches to the electromechanical transient model when the single-line-to-ground fault occurs at 12:00 p.m. The multi-time-scale tool switches back to the steady-state model when a steady-state condition is reached. The steady-state and transient voltages at the PV are presented in Figure 11.

The variation of the steady-state voltages at the PV between 6:00 a.m. and 8:00 a.m., between 8:00 a.m. and 4:00 p.m. and between 4:00 p.m. and 6:00 p.m. (Figure 11a) is caused by the change of the solar irradiance. From 12:00 a.m. to 6:00 a.m. and from 6:00 p.m. to 12:00 a.m., the solar irradiance is zero, so the steady-state voltages at the PV are flat. When the capacitor is turned off (8:00 a.m.), the steady-state voltages decrease. However, when the capacitor is turned on (4:00 p.m.), the voltages at the PV increase. According to Figure 11b, after the capacitor bank is de-energized at 8:00 a.m., the voltage magnitudes at the PV slightly drop. No voltage transient oscillations occur in this case. When the single-line-to-ground fault occurs on Phase A of the high voltage side of the transformer, Phase A and B voltages at the PV suddenly drop as illustrated in Figure 11c. Phase C voltage at the PV, however, slightly rises. The PV voltages return to their initial values after the temporary fault is cleared. After the capacitor bank is energized at 4:00 p.m., the electromagnetic transient voltage 
oscillation occurs as described in Figure 11d. The transient voltage oscillation disappears after a few cycles. The PV voltage magnitudes slightly increase after the capacitor bank is turned on.

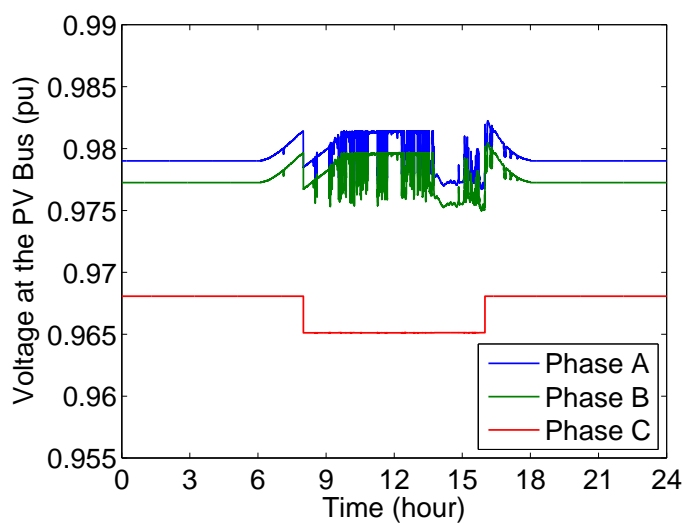

(a)

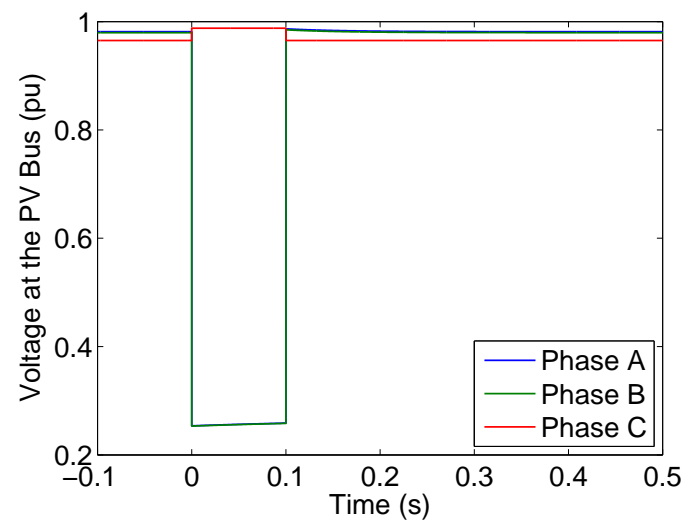

(c)

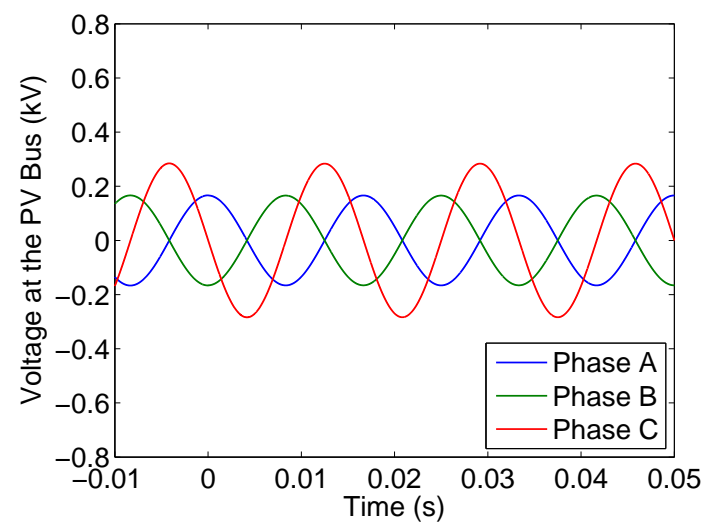

(b)

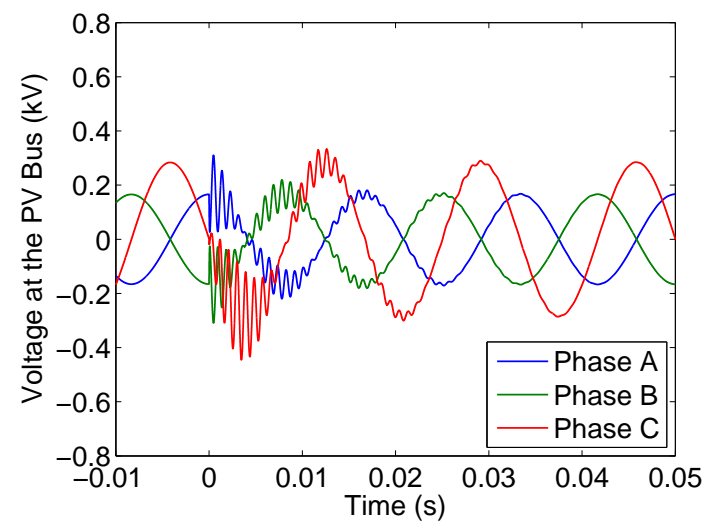

(d)

Figure 11. Voltages at the PV for Case 4. (a) Steady-state voltages at the PV; (b) transient voltages at the PV when the capacitor bank is turned off at 8:00 a.m.; (c) transient voltages at the PV when the fault occurs at 12:00 p.m.; (d) transient voltages at the PV when the capacitor bank is turned on at 4:00 p.m.

\section{Conclusions}

This paper develops an integrated multi-time-scale simulation tool for distribution circuits designed specifically for applications in wind turbine and PV analysis. The proposed multi-time-scale simulation tool consists of steady-state, electromechanical transient and electromagnetic transient models of voltage sources, distribution lines, transformers, loads, capacitor banks, wind turbines and PVs. The novel contribution of this proposed tool is the ability to switch from one single-time-scale model to another single-time-scale model when power system phenomena with different time scales occur. Therefore, this tool is capable of performing a long-term simulation involving power system phenomena spreading across time scales. Because the proposed tool employs models of distribution circuits with all three phases represented, the tool is able to analyze distribution circuits operating in unbalanced conditions due to single-phase loads and single-phase circuits in the systems.

Author Contributions: Pisitpol Chirapongsananurak and Surya Santoso collaboratively developed the idea, problem formulation, and test framework; Pisitpol Chirapongsananurak developed the models, performed the simulations, and wrote the paper; Surya Santoso helped with the analysis and paper writing.

Conflicts of Interest: The authors declare no conflict of interest. 


\section{References}

1. Thomson, M.; Infield, D.G. Impact of Widespread Photovoltaics Generation on Distribution Systems. IET Renew. Power Gener. 2007, 1, 33-40.

2. Dugan, R.C.; McGranaghan, M.F.; Santoso, S.; Beaty, H.W. Electrical Power Systems Quality, 3rd ed.; McGraw-Hill: New York, NY, USA, 2012.

3. Lubosny, Z. Wind Turbine Operation in Electric Power Systems; Springer: Berlin, Germany, 2003.

4. Meegahapola, L.; Fox, B.; Flynn, D. Flicker Mitigation Strategy for DFIGs during Variable Wind Conditions. In Proceedings of the 2010 IEEE Power and Energy Society General Meeting, Detroit, MI, USA, 24-29 July 2010; pp. 1-8.

5. Cipcigan, L.M.; Taylor, P.C. Investigation of the Reverse Power Flow Requirements of High Penetrations of Small-Scale Embedded Generation. IET Renew. Power Gener. 2007, 1, 160-166.

6. Howard, D.F.; Habetler, T.G.; Harley, R.G. Improved Sequence Network Model of Wind Turbine Generators for Short-Circuit Studies. IEEE Trans. Energy Convers. 2012, 27, 968-977.

7. Morren, J.; de Haan, S.W.H. Short-Circuit Current of Wind Turbines with Doubly Fed Induction Generator. IEEE Trans. Energy Convers. 2007, 22, 174-180.

8. Bradt, M.; Badrzadeh, B.; Camm, E.H.; Mueller, D.; Schoene, J.; Siebert, T.; Smith, T.; Starke, M.; Walling, R. Harmonics and Resonance Issues in Wind Power Plants. In Proceedings of the 2011 IEEE Power and Energy Society General Meeting, Detroit, MI, USA, 24-28 July 2011; pp. 1-8.

9. Tsai, J.; Tan, K. H APF Harmonic Mitigation Technique for PMSG Wind Energy Conversion System. In Proceedings of the 2007 Australasian Universities Power Engineering Conference, Perth, Australia, 9-12 December 2007; pp. 1-6.

10. Li, H.; Singhvi, V.; Maitra, A.; Rajagopalan, S.; Enayati, B.; Santoso, S.; Patterson, R. Analysis of PV Generation Impacts on Voltage Imbalance and on Voltage Regulation in Distribution Networks. In Proceedings of the 2014 IEEE Power and Energy Society General Meeting, National Harbor, MD, USA, 27-31 July 2014; pp. 1-5.

11. Wang, P.; Gao, Z.; Tjernberg, L.B. Operational Adequacy Studies of Power Systems with Wind Farms and Energy Storages. IEEE Trans. Power Syst. 2012, 27, 2377-2384.

12. Eftekharnejad, S.; Vittal, V.; Heydt, G.T.; Keel, B.; Loehr, J. Impact of Increased Penetration of Photovoltaic Generation on Power Systems. IEEE Trans. Power Syst. 2013, 28, 893-901.

13. Eftekharnejad, S.; Vittal, V.; Heydt, G.T.; Keel, B.; Loehr, J. Small Signal Stability Assessment of Power Systems with Increased Penetration of Photovoltaic Generation: A Case Study. IEEE Trans. Power Syst. 2013, 28, 893-901.

14. Coogan, K.; Reno, M.J.; Grijalva, S.; Broderick, R.J. Locational Dependence of PV Hosting Capacity Correlated with Feeder Load. In Proceedings of the 2014 IEEE PES Transmission and Distribution Conference and Exposition, Chicago, IL, USA, 14-17 April 2014; pp. 1-5.

15. Hoke, A.; Butler, R.; Hambrick, J.; Kroposki, B. Steady-State Analysis of Maximum Photovoltaic Penetration Levels on Typical Distribution Feeders. IEEE Trans. Sustain. Energy 2013, 4, 350-357.

16. Margaris, I.D.; Mantzaris, J.C.; Karystianos, M.E.; Tsouchnikas, A.I.; Vournas, C.D.; Hatziargyriou, N.D.; Vitellas, I.C. Methods for Evaluating Penetration Levels of Wind Generation in Autonomous Systems. In Proceedings of the 2009 IEEE Bucharest Power Tech Conference, Bucharest, Romania, 28 June-2 July 2009; pp. 1-7.

17. Fazeli, M.; Asher, G.M.; Klumpner, C.; Yao, L. Novel Integration of DFIG-Based Wind Generators within Microgrids. IEEE Trans. Energy Convers. 2011, 26, 840-850.

18. Jahangiri, P.; Aliprantis, D.C. Distributed Volt/Var Control by PV Inverters. IEEE Trans. Power Syst. 2013, $28,3429-3439$.

19. Chowdhury, B.H.; Ma, H.T.; Ardeshna, N. The Challenge of Operating Wind Power Plants within a Microgrid Framework. In Proceedings of the 2010 Power and Energy Conference at Illinois, Urbana, IL, USA, 12-13 February 2010; pp. 93-98.

20. Fazeli, M.; Asher, G.M.; Klumpner, C.; Yao, L. Novel Integration of Wind Generator-Energy Storage Systems within Microgrids. IEEE Trans. Smart Grid 2012, 3, 728-737.

21. Strachan, N.P.W.; Jovcic, D. Dynamic Modelling, Simulation and Analysis of an Offshore Variable-Speed Directly-Driven Permanent-Magnet Wind Energy Conversion and Storage System (WECSS). In Proceedings of the OCEANS 2007-Europe, Aberdeen, UK, 18-21 June 2007; pp. 1-6. 
22. Ahshan, R.; Iqbal, M.T.; Mann, G.K.I.; Quaicoe, J.E. Micro-Grid System Based on Renewable Power Generation Units. In Proceedings of the 2010 23rd Canadian Conference on Electrical and Computer Engineering, Calgary, AB, Canada, 2-5 May 2010; pp. 1-4.

23. Fadaeinedjad, R.; Moschopoulos, G.; Moallem, M. Using STATCOM to Mitigate Voltage Fluctuations due to Aerodynamic Aspects of Wind Turbines. In Proceedings of the 2008 IEEE Power Electronics Specialists Conference, Rhodes, Greece, 15-19 June 2008; pp. 3648-3654.

24. Jamshidi, A.; Ghahderijani, M.M.; Barakati, S.M. Power Quality Improvement in Stand-Alone Microgrid Including Fixed-Speed Wind Farm: Role of Dynamic Voltage Restorer. In Proceedings of the 2012 11th International Conference on Environment and Electrical Engineering, Venice, Italy, 18-25 May 2012; pp. 206-211.

25. Watson, N.R.; Arrillaga, J. Power Systems Electromagnetic Transients Simulation; The Institution of Electrical Engineers: London, UK, 2003.

26. Dommel, H.W. Digital Computer Solution of Electromagnetic Transients in Single- and Multiphase Networks. IEEE Trans. Power Appar. Syst. 1969, 88, 388-399.

27. Grainger, J.J.; Stevenson, Jr., W.D. Power System Analysis; McGraw-Hill: New York, NY, USA, 1994.

28. Masters, G.M. Renewable and Efficient Electric Power Systems, 2nd ed.; Wiley: Hoboken, NJ, USA, 2013.

29. Patel, M.R. Wind and Solar Power Systems: Design, Analysis, and Operation, 2nd ed.; CRC Press: Boca Raton, FL, USA, 2006.

30. Wu, B.; Lang, Y.; Zargari, N.; Kouro, S. Power Conversion and Control of Wind Energy Systems; Wiley: Hoboken, NJ, USA, 2011.

31. IEEE PES DSASC Test Feeder Working Group. Distribution Test Feeders. Available online: http://ewh.ieee. org/soc/pes/dsacom/testfeeders/ (accessed on 9 February 2017).

32. National Renewable Energy Laboratory. Western Wind Resources Dataset. Available online: http:/ / wind. nrel.gov/Web_nrel/ (accessed on 9 February 2017).

33. Chirapongsananurak, P.; Santoso, S. Distribution System Multi-Domain Simulation Tool for Wind Power Analysis. In Proceedings of the 2016 IEEE Power and Energy Society General Meeting, Boston, MA, USA, 17-21 July 2016; pp. 1-5.

34. Electric Power Research Institute. Smart Grid Resource Center: Simulation Tool-OpenDSS. Available online: http:/ / smartgrid.epri.com/SimulationTool.aspx (accessed on 9 February 2017).

(C) 2017 by the authors. Licensee MDPI, Basel, Switzerland. This article is an open access article distributed under the terms and conditions of the Creative Commons Attribution (CC BY) license (http:/ / creativecommons.org/licenses/by/4.0/). 